\title{
Buchbesprechungen
}

\section{Sammelbesprechung „Entstehung und Verfall internationaler Ordnung“}

Besprochen von Prof. em. Dr. Leo Bamberger, Senior non-resident fellow, ISPK, E-Mail: bamberger@ispk.uni-kiel.de

https://doi.org/10.1515/sirius-2020-1015

Die Erosion der in der Nachkriegszeit entwickelten internationalen liberalen, westlichen Ordnung ist heute offenkundig. Dieses Thema ist nicht neu und wurde schon vor mehr als zehn Jahren angesichts der Streitigkeiten innerhalb der westlichen Welt über die Fortführung und Verteidigung der internationalen Ordnung angesprochen. ${ }^{1}$ Heute sind es vor allem der Aufstieg Chinas und der Revisionismus Russlands sowie der Rückzug der Trump-Administration aus ihrer weltpolitischen Verantwortung, die Sorge bereiten. Die entscheidenden Fragen sind dabei:

1. Was ist und was konstituiert internationale Ordnung und was bewirkt sie?

2. Wie ist die derzeit gefährdete liberale westliche Ordnung entstanden und was waren und was sind ihre konstitutiven Elemente?

3. Was hat den Zerfall dieser internationalen Ordnung in den vergangenen Jahren bewirkt?

4. Was werden die absehbaren Konsequenzen des derzeitigen Ordnungsverfalls sein?

In der internationalen wissenschaftlichen Diskussion gibt es in der Auseinandersetzung mit der Thematik „liberale internationale Ordnung “ zwei unterschiedliche Ansätze. In dem ersten Ansatz wird das Thema theoretisch und deduktiv aufgegriffen. Ausgangspunkt ist die Frage, was eine liberale internationale Ordnung ausmacht und welche prinzipiellen Elemente eine derartige Ordnung haben sollte um wirksam zu sein. In der Regel gehen derartige Debatten auf Klassiker der Philosophie (zumeist auf Immanuel Kants Schrift zum Ewigen Frieden) zurück und versuchen anhand neuerer Entwicklungen präskriptive Vorschläge zu machen. Je breiter der Teilnehmerkreis an derartigen Debatten ist, desto vielfältiger werden allerdings die Antworten und umso diffuser die daraus abgeleiteten Empfehlungen. ${ }^{2}$ Oder sie führen dazu, dass auf einer relativ hohen Abstraktionsebene „wenn-dann“ Aussagen oder „sowohl-als-auch“ Behauptungen dominieren und

1 Krause 2007 und Krause 2017.

2 Council on Foreign Relations 2019.
Dilemmata und Paradoxien aufgezeigt werden, die am Ende die Leser eher unschlüssig zurückgelassen. ${ }^{3}$

Die andere wissenschaftliche Vorgehensweise besteht darin, real existierende regionale oder überregionale Beispiele internationaler Ordnungsbildung empirisch $\mathrm{zu}$ untersuchen, ihre Stärken und Schwächen herauszuarbeiten und zu fragen, was die Ordnung gefährdet oder stabilisiert. Die liberale internationale Ordnung ist dabei nur eine unter vielen Ordnungsbildungen der Geschichte, aber sie verdient natürlich die meiste Aufmerksamkeit, weil sie die heutige Zeit strukturiert hat. Diese Art des wissenschaftlichen Vorgehens wurde für die Politikwissenschaft durch die Englische Schule begründet, welche in den 50er und 60er Jahren des 20. Jahrhunderts entstand. ${ }^{4}$ Theoretischer Ausgangspunkt der Englischen Schule war die Annahme, dass sich trotz internationaler Anarchie ${ }^{5}$ immer wieder Muster der Kooperation zwischen Staaten (und auch nichtstaatlichen Akteuren) herausgebildet haben bzw. bilden und dass es darauf ankommt, dass diese Kooperation gefördert und stabilisiert sowie gegen Erosion geschützt wird. ${ }^{6}$ Internationale Ordnung besteht für die Englische Schule aus Arrangements und Regeln, die Staaten untereinander treffen, um die negativen Folgen der grundsätzlich bestehenden zwischenstaatlichen Anarchie abzumildern oder einzuhegen. Laut Hedley Bull, einem der führenden Vertreter der Englischen Schule, ist jegliche internationale Ordnung eine Form der Gesellschaftsbildung unter Bedingungen von Anarchie. ${ }^{7}$ Auch der frühere Harvard Professor und US-Außenminister Henry Kissinger hat sich - von der realistischen Schule her kommend - intensiv mit Fragen der empirischen Erforschung internationaler Ordnung befasst. Während das Hauptaugenmerk Kissingers auf der Frage lag, wie Staaten trotz grundsätzlich unterschiedlicher Ziele und machtpolitischer Gegnerschaft Regeln finden, die ein gegenseitiges Auskommen ermöglichen, ${ }^{8}$ befasst sich die Englische Schule darüber hinaus auch sehr breit mit imperialen oder hegemonialen Ordnungen einzelner Großmächte. ${ }^{9}$ Letzteres ist auch das beherrschende Thema einer Reihe von Autoren, die aus der institutionalis-

\footnotetext{
3 Kundnai 2017

4 Wight 1960.

5 Mit diesem Begriff wird der Grundzustand der internationalen Beziehungen beschrieben, die Abwesenheit einer zentralen, regelnden Institution.

6 Vgl. ausführlicher zur Englischen Schule Krause 2015, Dunne 1998, Buzan 2004.

7 Bull 1977.

8 Kissinger 1962, Kissinger 1995, Kissinger 2014.

9 Watson 1992.
} 
tischen Schule kommen und sich mit hegemonialer Stabilität auseinandersetzten. ${ }^{10}$ Eine weitere Denkrichtung geht von Ordnungsbildung als einem Grundelement menschlicher Gesellschaften aus und versucht die Bedeutung von Staatsbildung wie von internationaler Gesellschaftsbildung als Teil eines Kontinuums der Reduzierung von Unsicherheit zu begreifen. ${ }^{11}$

In den vergangenen Jahrzehnten sind eine Vielzahl von Büchern erschienen, die - auch wenn sie nicht explizit auf die Englische Schule oder auf Kissinger Bezug nehmen - sich mit der empirischen Realität von internationaler Ordnungsbildung befassen und versuchen daraus Schlussfolgerungen $\mathrm{zu}$ ziehen, die politisch verwertbare Aussagen erlauben. In der Regel befassten sich diese Arbeiten mit vergangenen Friedensverträgen und den durch sie geschaffenen Ordnungen. Dazu gehören der Westfälische Frieden, die Friedensschlüsse von Utrecht und Rastatt, der Wiener Kongress und das Europäische Konzert, die Gründung des Völkerbundes und dessen Scheitern sowie die Schaffung einer liberalen internationalen Ordnung nach dem Ende des Zweiten Weltkrieges und nach dem Ende des Kalten Krieges. Aber auch regionale Ordnungen außerhalb Europas wurden angesprochen.

Im Folgenden werden mehrere Bücher der vergangenen fünf Jahre vorgestellt, die diese Tradition fortführen und sich mit internationaler Ordnung generell und der Krise der westlichen Ordnung im Besonderen befassen. Am Ende des Überblicks wird versucht, die vier oben genannten Fragen vor dem Hintergrund der hier aufgeführten Literatur zu beantworten.

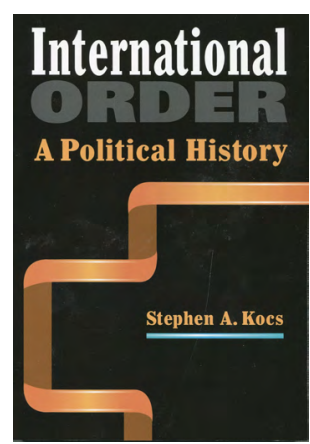

Stephen A. Kocs: International Order. A Political History. Boulder, Col.: Lynne Rienner Publishers 2019, 260 Seiten Um die Probleme der internationalen Ordnungsbildung $\mathrm{zu}$ verstehen, bedarf es seriöser Darstellungen dessen, was internationale Ordnung ist und welche historischen
Erfahrungen mit der Bildung und dem Verfall internationaler Ordnung gemacht worden sind. Von daher wird das vorliegende Buch von Stephen Kocs als Erstes besprochen. Es vermittelt eine gut lesbare und übersichtliche Einführung in die Geschichte internationaler Ordnungsbildung in Europa seit dem Mittelalter. Der Verfasser ist Associate Professor für Politikwissenschaft am Jesuiten-College Holy Cross in Worcester im US-Bundesstaat Massachusetts.

Der Autor lehnt sich bei der Definition von ,internationaler Ordnung“ an Schriften der Englischen Schule und an Kissinger an. Internationale Ordnung kann laut Kocs entweder dadurch zustande kommen, dass alle relevanten Mächte sich darauf verständigen bestimmte Regeln zu achten, die ihnen Restriktionen auferlegen, oder sie kann darin bestehen, dass die mächtigeren Nationen sich selber untereinander Regeln des Koexistierens geben und schwächeren Staaten ihre Regeln aufdrängen (S. 5). Das ist eine etwas knappe und nicht besonders präzise Definition, die im Buch immer wieder dafür sorgt, dass manches, was der Verfasser als internationale Ordnung ansieht, nicht immer überzeugend begründet wird. In der englischen Schule wird mit einem differenzierteren Begriff von Ordnung gearbeitet. $^{12}$

Das Kernargument des Buches ist, dass sich in Europa internationale Ordnung in drei Phasen herausgebildet habe: Im späten Mittelalter habe es einen Satz gemeinsam für wichtig gehaltener christlicher Werte gegeben, die die Grundlage für einige implizite Formen der Zurückhaltung der Herrscher untereinander und gegenüber ihren Untertanen abgegeben hätten. Die Institution der katholischen Kirche mit dem Zentrum des Vatikans habe diese Werte am Leben gehalten. Mit dem 18. Jahrhundert sei die Macht der Kirche zu schwach gewesen, um diese Ordnung aufrecht zu erhalten. Sie sei durch eine Ordnung der Machtbalance abgelöst worden, bei der die weltlichen Mächte pragmatische Wege gefunden hätten, einerseits ihre Interessen zu verfolgen, andererseits aber die Machtkämpfe nicht zu extrem ausarten lassen. Besonders nach blutigen Kriegen sei immer wieder versucht worden, ein Miteinander $\mathrm{zu}$ organisieren, welches die Wiederholung großer Kriege vermeiden sollte. Der Wiener Kongress von 1814/15 sei der Höhepunkt dieser Entwicklung gewesen. Mit dem Ersten Weltkrieg sei dann die Zeit der liberalen Ordnungsbildung angefangen, bei der versucht worden sei universelle Regelungen zur Friedenswahrung aufzustellen, Demokratie zu verbreiten und den internationalen Handel zu liberalisieren. Das Buch stellt ausführlich alle drei Phasen der Ordnungsbildung heraus und der Autor fasst die Ergebnisse

12 Watson 1992.

10 Ikenberry 2001, Ikenberry 2006, Ikenberry 2011.

11 Fukuyama 2015, Bobbitt 2002. 
im Abschlusskapitel zusammen, in dem auch auf die aktuellen Entwicklungen eingegangen wird.

Die Systematisierung, die Kocs vornimmt, ist nicht grundsätzlich falsch. Nur skizziert er die historischen Entwicklungen mit einem sehr groben Pinselstrich und präsentiert Entwicklungslinien, die beim näheren Hinschauen dann doch nicht so eindeutig sind. Das ist ein Fehler, den man oft bei Büchern findet, die versuchen, große Linien über die Jahrhunderte oder gar Jahrtausende zu ziehen. Dieses Buch ist bestimmt für Seminare an Universitäten hilfreich, aber es fehlt der Sinn für die Komplexität und politische Widersprüchlichkeit von Prozessen der Ordnungsbildung (und auch deren Verfall). Die jüngste Entwicklung, d.h. der Verfall der liberalen internationalen Ordnung wird auch vom Autor registriert und kommentiert. Dabei wird erkennbar, dass Stephen Kocs die Dramatik der derzeitigen Entwicklungen nur partiell reflektiert. Die internationale Politik der kommenden Jahre, so schreibt er, werde „etwas weniger“ durch liberale Prinzipien und „etwas mehr“ durch Machtpolitik bestimmt sein (S. 234). Wenn das so wäre, wäre das schön.

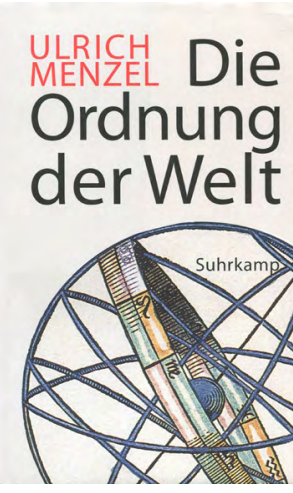

\section{Ulrich Menzel: Die Ordnung der Welt. Imperium oder Hegemonie in der Hierarchie der Staatenwelt. Berlin: Suhrkamp Verlag 2015, 1229 Seiten}

Deutlich anspruchsvoller als die Studie von Kocs ist das Buch von Ulrich Menzel, der bis zu seiner Emeritierung im Jahr 2015 Professor für Politikwissenschaft an der Technischen Universität Braunschweig war. Mit mehr als 1.200 Seiten und einem Gewicht von 1,2 Kg legt er ein großes Opus vor, gleichsam die Abschluss-Symphonie eines produktiven wissenschaftlichen Schaffens, welches eine Vielzahl von Ländern und Geschichtsperioden umfasst. So wie die Vertreter der Englischen Schule geht Menzel davon aus, dass die etablierten Theorien das, was die Welt im Innersten zusammenhält, nicht adäquat erfassen. Insbesondere stört ihn, dass viele Vertreter der realistischen
Theorieschule nicht über die Beschwörung der internationalen Anarchie hinausdenken würden (mit internationaler Anarchie ist, wie oben erwähnt, die Abwesenheit einer übergeordneten, regelnden Instanz gemeint). Anarchie sei zwar tatsächlich der Ausgangszustand der internationalen Politik. Aber, weil dies bekannt ist und weil die Furcht vor den negativen Konsequenzen der Anarchie groß ist, gäbe es seit Jahrhunderten Bemühungen, Anarchie abzumildern.

Die Anarchieproblematik könne, so Menzel, abgemildert werden durch die Bereitstellung internationaler öffentlicher Güter. Öffentliche Güter sind die Sicherheit gegenüber externen Bedrohungen, die Sicherheit der Seefahrt und die Schaffung von Bedingungen, unter denen Handel und Austausch zwischen Menschen und ganzen Gesellschaften stattfindet. Seine Ausgangsthese ist, dass in der Regel nur Großmächte in der Lage sind, allein oder maßgeblich für internationale Ordnung zu sorgen (S. 36). Kleinere Mächte wären nur in Ausnahmefällen und begrenzt dazu in der Lage. Es gäbe zwei Arten von Großmächten: solche die Imperien bilden würden und solche, die eine hegemoniale Ordnung herstellen und bewahren. „Imperien,“ so Menzel, „setzen immer den Einsatz von Gewalt, die Eroberung zur Durchsetzung eines Herrschaftsverhältnisses voraus“ (S. 39). Hegemonie hingegen bedeutet, dass eine Großmacht sich zum Führer einer Koalition von Staaten macht, die an der Herstellung einer internationalen Ordnung interessiert und bereit sind, diese Führung zu akzeptieren.

In einer hegemonialen internationalen Ordnung schaffe der Hegemon Sicherheit im militärischen und Stabilität im ökonomischen Bereich. Er wende dafür entsprechende Ressourcen auf und akzeptiere in der Regel, dass andere Staaten sich als free rider betätigten. Manchmal binde er auch Partnerstaaten in einem begrenzten Rahmen in dieses System ein, welches prinzipiell offen ist. Auch Imperien bieten nach Menzel militärische Sicherheit und wirtschaftliche Stabilität. Diese sei aber nur zum Preis der Unterwerfung zu haben und sie betreffe auch nur die Staaten, die Teil des Imperiums sind oder sich diesem unterwerfen. Die Finanzierung eines Imperiums erfolge durch Tributleistung, Ausplünderung, zwangsweise Rekrutierung von Arbeitskräften und Soldaten.

Das primäre Motiv zur Errichtung einer internationalen Ordnung sei die Aufhebung der Anarchie der Staatenwelt. Sie würde dem Hegemon den größten Nutzen verschaffen, aber auch den anderen Staaten die Möglichkeit verschaffen öffentliche Güter zu genießen, die anderenfalls nicht zu haben wären. Primäres Motiv zur Imperiumsbildung hingegen sei die Aufbringung von Tributleistungen, um dessen weitere Expansion zu finanzieren (S. 43). 
Die Leistungsfähigkeit einer hegemonialen Ordnung sei dann erreicht, wenn die Leistungsfähigkeit des Hegemons nachlässt und die Akzeptanz der von ihm geschaffenen Ordnung abnimmt. Imperiale Mächte zeichneten sich dadurch aus, dass sie primär auf militärische Macht setzen und die Interessen anderer missachten, hegemoniale Mächte würden eine umfassende Überlegenheit in wissenschaftlich-technischer, wirtschaftlicher, politischer, militärischer und kultureller Hinsicht aufweisen (S. 47). Hegemoniale Systeme seien offene Systeme ohne feste Grenzen mit variabler Reichweite, Imperien hingegen wären immer begrenzt und darauf bedacht ihre Grenzen zu sichern bzw. zu erweitern. Hegemonialmächte seien, so Menzel, primär Seemächte, imperiale Mächte hingegen Landmächte. Nur Seemächte würden es vermögen, dass Ströme von Waren und Menschen in sicherer Umgebung stattfinden können.

Ob eine Großmacht sich entscheidet als Hegemon oder als Imperium sein internationales Umfeld (oder eine Großregion) zu gestalten (oder gar weltweit zu wirken), hänge von dem politischen Willen seiner Führung aber auch von seinem politischen System ab. Staaten wie die USA und China hätten in ihrer Geschichte langanhaltende Perioden des Isolationismus gehabt. Die Entscheidung der USA nach dem Zweiten Weltkrieg eine internationale Ordnung im Sinne einer wohlmeinenden Hegemonie zu errichten, sei aus der Besonderheit der damaligen internationalen Lage (insbesondere die Erfahrung der zwei Weltkriege) geboren worden. Ob die USA diese Rolle beibehielten, sei offen. Auch, welchen Kurs sie alternativ einschlagen würden: den des Rückfalls in Nationalismus und Isolationismus, oder den des Übergangs zu einer eher imperialen Ordnung.

Die theoretischen Überlegungen zu Hegemonie und internationaler Ordnung sowie zu Imperien passen gut zu dem Gedankengebäude der Englischen Schule. Ulrich Menzel scheint diese Schule aber nicht zu kennen, denn die einschlägigen Bücher tauchen in seiner 72 Seiten langen Literaturliste nicht auf. Insofern hat er mit seinem theoretischen Ansatz quasi „das Rad neu erfunden“, aber das ist ihm sehr gut gelungen. Sein Ansatz hilft die Entstehung und die Entwicklung der neuesten Zeit zu verstehen. Von den 14 historischen Fallstudien, die dem Theorieteil folgen (und die das Buch so unendlich umfangreich werden lassen) sei hier nur kurz diejenige über die USA als Hegemon erwähnt. Der Verfasser unterscheidet zwischen zwei Phasen der amerikanischen Hegemonie: die Zeit zwischen 1898 und 1990 und die Periode nach 1990 bis heute. Die Logik dieser Unterscheidung leuchtet dem Rezensenten nicht ein und macht wenig Sinn. Auch ist die Darstellung der Entwicklung der internationalen Ordnung nach 1945 unter US-Führung doch etwas zu USA-zentrisch, die
Rolle der Europäer bleibt weitgehend ausgeblendet. Seine Prognose, wonach sich an der Hegemonie der USA erst einmal nichts wirklich Wesentliches ändern wird (S. 1014) wirkt angesichts der Entwicklungen nach Erscheinen des Buches (insbesondere nach dem Amtsantritt Donald Trumps als 45. Präsident der USA) nicht sehr überzeugend. Dennoch ist es ein großartiges Buch, welches eine interessante Perspektive auf die letzten 1000 Jahre der Geschichte der internationalen Politik bietet, die sich positiv von vielen anderen Darstellungen abhebt.

Die nachfolgenden Bücher befassen sich mit der Frage, warum die westliche, liberale internationale Ordnung, die 1989 auf dem Vormarsch zu sein schien, heute einer Erosion unterliegt. $\mathrm{Zu}$ dieser Frage gibt es unterschiedliche Befunde, die im Folgenden vorgestellt werden.

\section{Michael Mandelbaum: The Rise and Fall of Peace on Earth. Oxford und New York: Oxford University Press 2019, 218 Seiten}

Das Buch von Michael Mandelbaum, Professor Emeritus an der Johns Hopkins School of Advanced International Studies (SAIS), befasst sich mit der Frage, warum der Friede heute beendet ist, der 1989 durch das Ende des Ost-West-Konfliktes eingeleitet wurde. Für Mandelbaum ist der Post-Cold War Peace bemerkenswert, weil er erstmals eine Periode einläutete, die durch die Abwesenheit strategischer Konkurrenz gekennzeichnet gewesen ist. Anders als die Beendigung früherer strategischer Konkurrenzbeziehungen sei dieser Friede nicht durch einen großen Friedensschluss oder ein Diktat der Sieger beendet worden, sondern durch die Übereinkunft unter den bisherigen Konfliktparteien die Konkurrenz zu beenden. Dieser Friede, der in erster Linie die Mitgliedstaaten von NATO und Warschauer Pakt umfasste, strahlte allerdings auch auf den Fernen Osten sowie auf den Nahen Osten aus. Sowohl in Ostasien wie im Nahen Osten war seinerzeit die Erwartung groß, dass auch dort die strategischen Konfrontationen aufhören würden. Zwischen Israel und den Arabern gab 
es Zeichen der Annäherung und China beschritt den Weg der Wirtschaftsreformen, der ohne eine Integration in das westliche dominierte Welthandelssystem und ohne die Gewährung individueller und politischer Freiheiten unvorstellbar erschien. All diese Hoffnungen seien heute zerschlagen und der Verfasser versucht Antworten auf die Frage zu finden, was den Frieden von 1989 möglich gemacht hat und warum dieser Friede heute zerstört ist.

Der friedliche Übergang 1989/1990 war für Mandelbaum durch den Kollaps des wissenschaftlichen Marxismus und der mit dieser Staatsphilosophie verbundenen kommunistischen Parteien verursacht, dem auf Seiten der westlichen Welt ein bereits existierendes und funktionierendes System der friedlichen und zivilen Ordnung gegenüberstand. Das System der westlichen Ordnung hatte sich als überlegen erwiesen und bestand aus drei Elementen: der US-amerikanischen Hegemonie (als unverzichtbare Führungsmacht), der Dominanz demokratischer Regierungssysteme (denn Demokratien neigen dazu den internen Modus des Verhandelns und der Suche nach Moderation $\mathrm{zu}$ externalisieren) und der Liberalisierung des Handels (die den Wohlstand geschaffen hat). Das westliche Ordnungssystem hätte auf Europa, Ostasien und den Mittleren Osten ausgedehnt werden können, wenn diese drei Bedingungen in allen drei genannten Regionen akzeptiert worden wären. Tatsächlich zeichnete sich in den 90er Jahren eine derartige Entwicklung ab, diese sei aber nach und nach abgebrochen, weil vor allem Russland, China und der Iran den Weg der Autokratie gegangen seien und sich der amerikanischen Hegemonie entgegengestellt hätten. Auf das Freihandelssystem hätte sich vor allem China eingelassen, aber auch nur unter Bruch vieler Regeln.

China, Russland und den Iran hätten, so Mandelbaum, aus primär innenpolitischen Gründen den Weg der Konfrontation mit dem Westen gesucht und somit den Frieden zerstört, der sich 1990 in allen drei Regionen abzuzeichnen begann. Russland werde durch eine kleptokratisch agierende Machtvertikale regiert, die innere Stabilisierung durch äußere Aggressivität und die Rückweisung jeglichen amerikanischen Hegemonieanspruchs sucht. In China sei die Lage grundsätzlich nicht anders. Hier halte die Kommunistische Partei unbeirrt und mit allen verfügbaren Machtmitteln an ihrem Machtmonopol fest und nutze zudem anti-westliche und nationalistische Narrative, um die eigenen Legitimationsdefizite auszugleichen, die durch Inkompetenz und Korruption verschlimmert werden. Zudem verfolge die chinesische Führung seit den 50er Jahren einen revisionistischen Kurs und versuche Grenzen zu Land wie zur See zu ihren Gunsten zu verändern. Im Iran sei die Revolution von 1979 gescheitert. Geblieben sei eine revolutionäre und korrupte Machtelite, die durch Expansion in der Region versuche von ihrem Misserfolg abzulenken. Allen drei Herausforderern sei gemein, dass eine Machtvertikale die staatlichen Institutionen überlagere und kontrolliere. Dadurch werde eine friedliche Entwicklung dieser Länder verhindert, sie werden zu einer Belastung für den Frieden in Europa, in Ostasien und im Mittleren Osten. Mandelbaum legt in seinen drei Hauptkapiteln für jede dieser Regionen den Zerfall der Friedensentwicklungen präzise dar. Im Mittleren Osten käme hinzu, dass es dort weder einen Trend in Richtung Demokratie noch ein Maß an Handelsliberalisierung gäbe, welches zu einer Wohlstandssteigerung beitragen würde.

Das von Mandelbaum beschriebene Konfliktmuster dürfte für die kommenden Jahre und Jahrzehnte ausschlaggebend sein. Es ist kein Konflikt - wie der Ost-WestKonflikt - über die grundlegende Richtungsentscheidung zwischen Kapitalismus und Sozialismus, sondern ein Konflikt zwischen einer (mittlerweile schwächelnden bzw. sich ihrer Rolle als Führungsmacht einer internationalen Ordnung unsicher werdenden) USA und den drei Herausforderern, die die westlich geprägte, regelbasierte Ordnung entweder ganz oder zumindest in großen Teilen zerstören wollen. Dabei muss auch mit dem zunehmenden Einsatz militärischer Gewalt gerechnet werden.

Mandelbaum fordert, dass die USA und ihre Verbündeten die Natur dieses Konfliktes begreifen und ihre Politik daran ausrichten. Er kritisiert die NATO-Erweiterung, weil diese das nationalistische Narrativ in Russland unterstützt habe. Er kritisiert die amerikanische Besetzung des Iraks im Jahre 2003, weil diese den wichtigsten Gegner des Iran ausgeschaltet und dem Iran den Weg in Richtung Mittelmeer geöffnet habe. Er kritisiert weiterhin den Abschluss des Atomabkommens (JCPOA) mit dem Iran im Sommer 2015, weil dieses nicht konsequent genug die Aggressivität des Iran gestoppt, sondern diese eher gefördert habe. Mandelbaum kritisiert auch, dass die Staaten des Westens viel zu lange hingenommen hätten, dass China die Regeln der WTO zu seinen Gunsten verletzt. Außerdem sei viel zu lang die militärische Gefahr unterschätzt worden, die sich aus den verschiedenen territorialen Ansprüchen Chinas ergäben, vor allem gegenüber Taiwan, welches heute eine funktionierende Demokratie sei. Für ihn scheint die EinChina-Politik der westlichen Staaten ein schwerer Fehler zu sein, denn diese ermutige China zu einer aggressiven, militärischen Politik gegenüber Taiwan.

Mandelbaum fordert vor allem, dass sich westliche Politik auf die Natur dieses Konfliktes einstellt und nicht $a d-h o c$ nach Lösungen sucht, die vorübergehende Ruhe bieten, aber die grundsätzliche Konfliktdynamik ignorier- 
ten. Folgt man Mandelbaums Analyse, dann ist davon auszugehen, dass die Aggressivität und Militanz Russlands, Chinas und des Iran nicht nachlassen wird und dass es dem Westen gelingen muss, ein neues außenpolitisches Instrumentarium $\mathrm{zu}$ entwickeln, welches mit den militärischen, ökonomischen und technologischen Herausforderungen Seitens dieser drei Staaten fertig wird und gleichzeitig die Demokratisierung fördert.

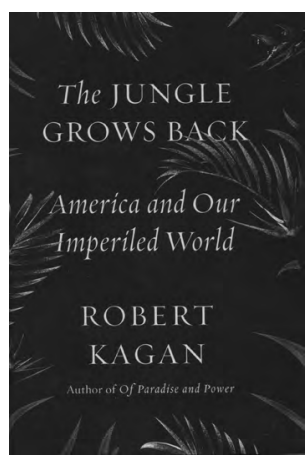

\section{Robert Kagan: The Jungle Grows Back. America and Our Imperiled World. New York: Alfred Knopf 2018, 180 Seiten}

Robert Kagan von der Brookings Institution hat ein kurzes, eher essay-artiges Buch mit einem knalligen Titel vorgelegt, welches international viel Aufsehen erregt hat, in Deutschland jedoch weitgehend unbeachtet geblieben ist. Leider ganz zu Unrecht, denn dieses Buch entwirft ein durchaus realistisches wie pessimistisches Bild der politischen Zukunft und stellt vor allem grundlegende Annahmen der deutschen Politik in Frage (wie die Suche nach strategischer Autonomie Europas, die Anwendbarkeit von Rezepten der Ostpolitik gegenüber Russland, die Politik des Festhaltens am Multilateralismus, den Optimismus, was die Zukunft der europäischen Integration betrifft). Vielleicht wird es auch gerade deswegen ignoriert. Wissenschaftlich gesehen bleibt es hinter den üblichen Standards zurück, aber es ist ja gerade der Charme eines wissenschaftlichen Essays, das mit ihm versucht wird in einer Frage weiter $\mathrm{zu}$ kommen, bei der die detaillierte Analyse von einzelnen Wirkfaktoren nicht weiterführt. Es ist gleichsam ein kühner Entwurf, der versucht größere Zusammenhänge zu verstehen.

Kagans Kernthese lautet: die Normalität der zwischenstaatlichen Politik ist seit bestimmt 5.000 Jahren der ewige Kampf jeder gegen jeden, die internationale Anarchie. Diese zu bändigen ist immer wieder in der Weltgeschichte durch regionale oder global wirkende Großmächte versucht worden - mit unterschiedlichen Resultaten. Eine aufsteigende, zivilisatorische Linie gibt es nicht, denn die schlimmsten Gewaltexzesse fanden im 20. Jahrhundert statt. Der letzte und erfolgreichste Versuch war die Schaffung einer liberalen internationalen Ordnung durch die USA nach Ende des Zweiten Weltkriegs. Dieser Schritt markierte eine Absage an die bis dahin verfolgte Politik der Isolation und war motiviert durch die Erfahrungen der beiden Weltkriege. Sie war konditioniert durch die Tatsache, dass die USA eine liberale Demokratie sind. Der liberale Charakter der internationalen Ordnung (die nicht universell ist, aber zumindest weltumspannend) sei durch folgende Eigenschaften charakterisiert: die Berücksichtigung der Interessen der anderen Akteure durch den Hegemon, die Orientierung an Menschenrechten und Handelsliberalisierung sowie die Verantwortung des Hegemons dafür, dass Frieden innerhalb der Zone der internationalen Ordnung bewahrt bleibt und auch von außen nicht gefährdet ist.

Diese internationale Ordnung funktioniert so lange, so Kagan, wie die USA weltweit die stärkste Wirtschafts- und Militärmacht aufbieten können und solange, wie ein überparteilicher politischer Konsens in Washington besteht, wonach es im übergeordneten Interesse der USA sei, diese Rolle weiterhin zu spielen. Tatsächlich würden wir uns derzeit in einer Phase befinden, wo zum einen der relative Machtvorsprung der USA vor anderen Mächten (vor allem China) schmilzt und zum anderen die politische Bereitschaft in den USA abnimmt weiterhin Verantwortung für die internationale Ordnung zu übernehmen. Kagan sieht diese Führungsrolle sowohl von Seiten der Demokraten wie der Republikaner gefährdet. In der Demokratischen Partei hätten diejenigen Stimmen an Kraft gewonnen, die vor allem militärische Interventionen grundsätzlich kritisch sehen (Imperialismusverdacht), in der Republikanischen Partei diejenigen, die forderten, dass die USA sich wie ein „normale“ Macht verhalten und primär ihre eigenen Interessen verfolgen sollten. Angesichts der verlustreichen und problembehafteten Interventionen in Afghanistan und dem Irak und den enormen Kosten dieser Unternehmungen sei dieser Widerstand nachvollziehbar, es sei aber, so Kagan, kurzsichtig zu glauben, dass ein Rückzug der USA aus weltpolitischer Verantwortung und die Rückkehr zu einer rein interessengeleiteten Außenpolitik im aufgeklärten Interesse der USA seien.

Für Kagan war schon die Außenpolitik der ObamaAdministration ganz klar von dem Bemühen gekennzeichnet gewesen, die Rolle als Garantiemacht der liberalen internationalen Ordnung zurück zu fahren. Das zögerliche und nachgiebige Verhalten im Syrien-Krieg und vor allem nach der russischen Intervention gegen die Ukraine hätten das deutlich gemacht. Hingegen sei sein pivot for Asia lediglich verbal erfolgt. Erst richtig schlimm 
sei es unter Trump gekommen, der die neue „Normalität“ der US-Außenpolitik verkörpere und Freund und Feind gleichermaßen mit seinen unberechenbaren Aktionen vor den Kopf stoße. Besonders im Nahen Osten sei zu sehen, wohin die Politik Obamas und Trumps geführt habe. Es sei zu einer Re-Anarchisierung der zwischenstaatlichen Politik gekommen, wo nur noch das Recht des Stärkeren zähle und die regionalen Mächte ihre Ziele brutal und rücksichtslos verfolgten. Das trifft für Russland, den Iran oder die Türkei ebenso zu wie für Saudi-Arabien oder Syrien.

Kagan arbeitet mit einer Metapher: Die Schaffung einer internationalen Ordnung durch eine Großmacht ist so etwas wie das Anlegen eines Gartens: dieser muss ständig gehegt und gepflegt werden, sonst holt sich die Natur den Garten zurück und die Regeln des Dschungels gelten erneut. Diese Metapher mag simpel klingen, aber es ist etwas dran an ihr. Sollten sich die USA weiter aus ihren internationalen Verpflichtungen zurückziehen, so sieht Kagan auch für Europa den Anbruch einer Zeit, in der wieder nationale Stereotypen vorherrschen und die alten Probleme auftauchen.

Gegen Ende versucht Kagan nicht ganz in Pessimismus zu verfallen. Er appelliert an seine Landsleute nicht den gefährlichen Weg des Rückzugs auf den amerikanischen Kontinent zu wagen, sondern wieder die Rolle des Garanten einer liberalen internationalen Ordnung zu spielen, die den amerikanischen Interessen weitaus mehr diene als Isolationismus oder die „Normalisierung“.

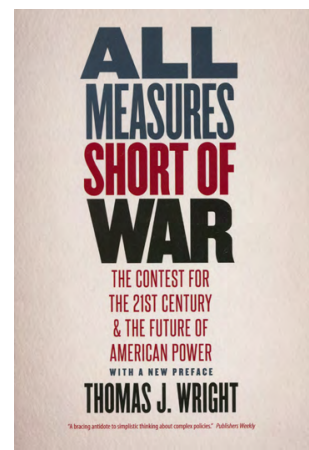

Thomas Wright: All Measures Short of War. The Contest for the Twenty-First Century and the Future of American Power. New Haven: Yale University Press 2017, 270 Seiten.

Das Buch von Thomas Wright von der Brookings Institution in Washington, D.C. setzt dort an, wo Mandelbaum und Kagan aufhören. Er konstatiert ebenso wie die zuvor genannten Autoren, dass die Zeit der von den USA geführ- ten und garantierten liberalen internationalen Ordnung vorbei sei und dass es darauf ankomme, sich auf eine Zeit einzustellen, in der strategische Konkurrenz und Rivalität unter Bedingungen fortlaufender Globalisierung die internationalen Beziehungen strukturieren werden. Seine Ausgangsfrage ist: wie muss man sich die künftige Natur der internationalen Beziehungen vorstellen? Und was bedeutet das für die amerikanische Außenpolitik?

Eine der Kernaussagen seines Buches ist, dass wir uns in einer Phase des Übergangs von einer geordneten und kooperativen Welt in eine solche befinden, in der Rivalität und Konkurrenz dominieren. Derartige Übergangsphasen, so Wright, seien dadurch charakterisiert, dass alle Akteure aus einer Situation der Verunsicherung darüber agieren, wie andere handeln, was sie selber bewirken können und wo die Risiken liegen. Das damit verbundene Maß an Unsicherheit könne zu krisenhaften Entwicklungen beitragen, bis irgendwann der Punkt erreicht sei, wo sich die wesentlichen Eckpunkte dessen abzeichneten, was möglich ist und was nicht.

Eine weitere Kernaussage ist, dass sich die USA und ihre Verbündeten darauf einstellen müssen, dass zwar einerseits alle Großmächte nukleare Kriege vermeiden wollen, dass aber unter der Ebene des Kernwaffenkrieges oder des offenen Krieges alle Großmächte heftig miteinander konkurrieren werden, auch unter Einsatz militärischer Mittel, hybrider Verfahren und ökonomischer Sanktionen. Von daher wird es für die USA und ihre Verbündeten wichtig sein, ihre diplomatischen und militärischen Instrumente neu zu justieren und vor allem als westliche Staatengemeinschaft enger beisammen zu stehen.

Wrights Empfehlung an die USA für die Phase nach Trump läuft darauf hinaus, dass alles vermieden werden muss, was auf eine Isolation der USA von ihren Verbündeten hinauslaufe. Die Rolle der westlichen Welt lasse sich nur dadurch stärken, dass in den drei Regionen Europa, Ostasien und dem Nahen Osten die USA und ihre westlichen Verbündeten (nicht nur die NATO-Mitglieder, sondern auch Japan, Südkorea, Australien und andere) eng zusammenarbeiten, um die Werte und Interessen der westlichen Staatengemeinschaft und der liberalen internationalen Ordnung so weit es geht aufrecht zu erhalten. 


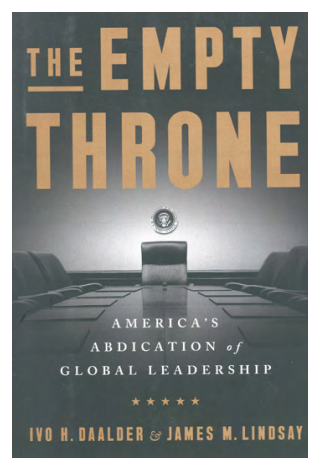

Ivo Daalder/James Lindsay: The Empty Throne. America's Abdication of Global Leadership. New York: Public Affairs 2018, 237 Seiten

Die USA waren für Jahrzehnte die treibende Kraft und der Garant der liberalen internationalen Ordnung. Seit Januar 2017 sitzt mit Donald Trump ein Präsident im Weißen Haus, dem diese Rolle der USA nicht nur gleichgültig ist, sondern der dezidiert eine Politik des America First betreibt und der den internationalen Multilateralismus (vor allem in der WTO, aber auch in anderen Bereichen wie der Klimapolitik) offen ablehnt und bestehende Bündnisse und strategische Allianzen in Frage stellt. Die Verfasser sind in der Szene wohl bekannt: Ivo Daalder ist Präsident des Chicago Council on Global Affairs, James Lindsay ist dort sein Stellvertreter. Beide hatten unter den demokratischen Präsidenten Bill Clinton und Barack Obama wichtige Regierungsämter eingenommen. Von daher überrascht nicht der kritische Ton der Analyse, die dennoch seriös bleibt.

Die Autoren zeichnen ein kritisch-nuanciertes Bild von der Entwicklung des Unternehmers und Medienstars Donald Trump zum Politiker und Präsidenten der USA. Sein Markenzeichen ist die Vorstellung, dass die Regierung von inkompetenten Politikern und Experten gesteuert wird und dass es eines kraftvollen und überlegenen Politikers wie ihm bedarf, der die Dinge wieder auf das richtige Maß zurückbringt, um den amerikanischen Wählern das $\mathrm{zu}$ geben, was sie verdienen. In mehreren, lesenswerten Kapiteln verfolgen sie die einzelnen Stationen der Trumpschen Außen-Sicherheits- und Außenhandelspolitik und gelangen zu teilweise vernichtenden Urteilen über dessen Bilanz. In keinem Feld habe er seine Ziele erreicht, vielmehr habe er Freunde und Gegner verunsichert und viel dazu beigetragen, dass andere Mächte - allen voran die VR China - immer einflussreicher würden.

Den größten Schaden der Politik Trumps sehen die Verfasser für die internationale Ordnung. Wir befinden uns in einer Welt, in der niemand mehr führt, so das Resümee der Autoren (S. 171). Für die Zukunft entwickeln die Verfasser drei Szenarien: entweder wird die internationale
Politik wieder wie im 19. Jahrhundert durch die Versuche der großen Mächte gekennzeichnet sein, sich gegenseitig Einflusszonen zu gewährleisten, die einigermaßen Frieden unter den großen Staaten versprechen, oder es wird die blanke Anarchie herrschen, bei der sich keiner der dazu befähigten Mächte in der Lage sieht für ein Mindestmaß an Ordnung zu sorgen. Es sei zwar vorstellbar, dass die europäischen Staaten eine gewisse Ordnungsrolle übernähmen, aber diese wären zu wenig mit effektiven Machtmitteln ausgestattet, um diese Aufgabe wahrzunehmen. Eher sehen die Verfasser die Volksrepublik China in der Lage, die internationale Ordnung zu gestalten, wenngleich sie auch hier große Fragezeichen ansetzen. Eine internationale Ordnung, die von China geprägt wäre, so Daalder und Lindsay, wäre allerdings eine Ordnung, die nicht den Interessen der USA entspräche. Diese Feststellung leuchtet eigentlich ein, allerdings scheint sie dem amerikanischen Präsidenten nicht vermittelbar zu sein.

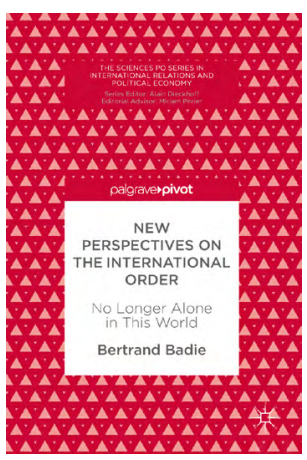

Bertrand Bardie: New Perspectives on the International Order. No Longer Alone in This World. London: Palgrave/ MacMillan 2016 (Sciences Po Series in International Relations and Political Economy), 140 Seiten

Das schon 2015 in französischer Sprache erschienene Buch des emeritierten Professors vom renommierten institut d'études politiques de Paris (Sciences Po) nimmt einen völlig anderen Ansatz zur Thematik „Internationale Ordnung“ als die Autoren aus dem angelsächsischen Bereich. Bardie ist stark in der Geschichte der internationalen Beziehungen des 19. Jahrhundert verankert. Sein Buch besteht aus sieben unterschiedlichen Kapiteln und einem kurzen abschließenden Teil. Die Kapitel sind mehr oder weniger allein stehende Essays, die nur wenig Bezug zueinander haben.

Eines der Hauptargumente von Bardie ist, dass die moderne internationale Ordnungsbildung mit dem Westfälischen Frieden (1648) einsetzt, der zum ersten Mal das von dem französischen Philosophen Jan Bodin ent- 
wickelte Prinzip der staatlichen Souveränität zum Leitmotiv erkoren habe. Kern des Souveränitätsprinzips sei die Anerkennung der territorialen Unversehrtheit souveräner Staaten durch die anderen souveränen Staaten und die damit einhergehende Legitimation moderner Staatlichkeit. Das andere Kernprinzip sei die absolute Willensfreiheit der souveränen Staaten und der sie dominierenden Herrscher. Aus diesem Dualismus sei immer wieder dann ein Widerspruch entstanden, wenn souveräne Herrscher es für richtig erachtet hätten, die Souveränität und Integrität anderer souveräner Akteure zu missachten. Von daher hätte die durch den Westfälischen Frieden geschaffene Ordnung nicht dazu getaugt, den zwischenstaatlichen Frieden zu wahren. Die einzige Ausnahme seien Arrangements unter den Großmächten, die darauf hinausliefen, untereinander Kriege zu vermeiden. Anlass sei immer die Befürchtung gewesen, dass Kriege eine unkontrollierbare Dynamik auslösen könnten, die die innenpolitische Stabilität in den großen Staaten hätte in Frage stellen können.

Besonders der Wiener Kongress war für ihn ein Beispiel dafür, wie aus innenpolitischen Stabilitätserwägungen heraus internationale Ordnungsbildung entstehen kann, die durchaus friedenssichernde Wirkung zeitige. Das Hauptproblem dieser Ordnungsbildung bestünde aber darin, dass es nur die wenigen Mächtigen der damaligen Welt waren, die diese Ordnung konstituiert und aufrechterhalten hätten. Der Wiener Kongress hätte seiner Meinung nach zwei Kernfehler enthalten: zum einen habe er die gesellschaftliche Entwicklung in den Ländern Europas weitgehend vernachlässigt bzw. als zu kontrollierendes Problem angesehen, zum anderen sei der oligarchische Charakter der Ordnungsbildung dafür verantwortlich gewesen, dass manche Mächte (wie etwa das Osmanische Reich) draußen vor blieben und sich als Herausforderer der bestehenden Ordnung betätigten. Beide Mängel hätten im 19. Jahrhundert dazu beigetragen, dass die positiven Elemente der Ordnungsbildung von 1815 verloren gegangen wären.

In der zweiten Hälfte des 20. Jahrhunderts hätte sich eine internationale Ordnung getragen von den westlichen Mächten herausgebildet, die zwar einerseits von dem Gedanken der Friedenssicherung und der Wohlstandsmehrung aller getragen war, die andererseits aber die gleichen Fehler aufweise wie die von 1815: sie ignoriere gesellschaftliche Entwicklungen und die würde Mächte wie Russland und China und vor allem die Staaten der sogenannten Dritten Welt ausgrenzen. Schon der Ost-WestKonflikt sei entstanden, weil die Westmächte es nicht verstanden hätten die Sowjetunion in die Ordnungsbildung einzubeziehen. Auch wäre die Dritte Welt von der Mitwirkung an der Ordnung ausgeschlossen geblieben. Nach dem Ende des Ost-West-Konflikts seien kurzfristig die USA zur hegemonialen Macht geworden, aber im Wesentlichen sei das Zusammenspiel der führenden westlichen Mächte (G7 und NATO) die treibende und tragende Kraft der internationalen Ordnungsbildung geblieben. Mit dem Wiedereintritt Russlands als Gegenmacht und dem Aufstieg Chinas zum globalen Herausforderer sowie dem zunehmenden Gewicht der Staaten Asiens in der Weltpolitik könne internationale Ordnungsbildung nicht mehr länger von der westlichen Staatengemeinschaft alleine getragen werden. Zudem hätten die westlichen Staaten die gesellschaftliche Entwicklung nicht gebührend berücksichtigt. Diese bestünde nicht nur aus der demographischen Schwerpunktverschiebung in Richtung Asien und Afrika, sondern vor allem in der Globalisierung und durch die von der digitalen Revolution getragenen Entwicklung in Richtung einer Weltgesellschaft.

Die Argumentation von Bardie leuchtet teilweise ein, es fehlen jedoch konkrete Hinweise darauf, wie sich der Verfasser die Neuordnung unter Vermeidung aller von ihm aufgezeigten oder vermuteten Fehler vorstellt. Hier bleibt das Buch unspezifisch und schweift in Allgemeines ab. Es ist leicht, den Westen generell und Frankreich im Besonderen der Arroganz und Ignoranz zu bezichtigen und die globale gesellschaftliche Entwicklung in groben Pinselstrichen unter Anführung vieler modischer Substantive und Eigenschaftsworte zu zeichnen. Daraus entsteht noch keine Vorstellung, wie es realiter anders aussehen könnte. Bardie fordert mehr Diplomatie und die Anerkennung des Andersseins. Das ist nichts Neues und bringt die Debatte um die Fortführung internationaler Ordnung kein Stück weiter.

Was Bardie übersieht ist, dass es außerhalb der westlichen Staatengemeinschaft bislang keine vergleichbare internationale Ordnungsbildung gegeben hat (auch nicht unter den Blockfreien), bzw. dort, wo es Ansätze gibt (wie bei der Afrikanischen Union) diese ohne massive westliche Unterstützung nie substantielle Fortschritte hätten machen können. Das, was Russland oder China an ordnungspolitischen Ansätzen vorweisen können, ist zumeist von einer Art, dass es bei den Nachbarn und anderen davon Betroffenen sehr bald negative Reaktionen hervorruft. Es ist schade, dass der Verfasser des Buches auf diese Fragen kaum eingeht, sondern sich in der Rolle des grundlegenden Kritikers westlicher Ordnungsbildung gefällt, der sich letztlich nur in nebulöse Kategorien verirrt. 


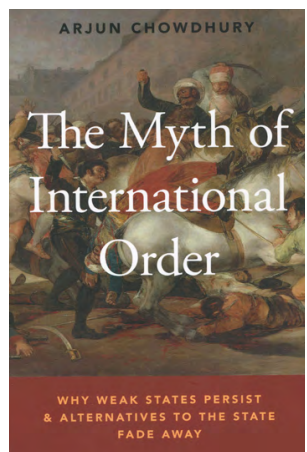

Arjun Chowdhury: The Myth of International Order. Why Weak States Persist and Alternatives to the State Fade Away. Oxford und New York: Oxford University Press 2018, 254 Seiten

Eine völlig andere Perspektive auf internationale Ordnung bietet das Buch von Arjun Chowdhury, der an der Universität von British Columbia in Kanada Politikwissenschaft lehrt. Chowdhury nimmt eine nicht-westliche Perspektive ein, schließt aber an die vorherrschende angelsächsische Denkschule an. Sein Ausgangspunkt ist die Annahme, dass die derzeitige liberale, westlich geprägte internationale Ordnung nur funktioniert, weil sie auf dem Engagement von funktionierenden Staaten beruht, die es geschafft haben ihren Bürgern ein hohes Maß an Sicherheit (vor allem Rechtssicherheit) und Wohlstand zu gewähren, gepaart mit Möglichkeiten der demokratischen Teilhabe und der Sicherung politischer Freiheit. Die Erwartung, dass sich die internationale Ordnung in dem Maße ausbreitet, wie dieses erfolgreiche Muster staatlicher Organisation in Ländern außerhalb des Westens erfolgreich nachgeahmt wird, ist nach Auffassung des Verfassers jedoch unrealistisch.

Ursache sei die Tatsache, dass die westlichen Staaten so wie wir sie heute kennen, das Produkt spezifischer historischer Umstände sind. Die Transformation zur heutigen Staatlichkeit sei die Folge von Jahrhunderten von Kriegen zwischen den europäischen Nationen, insbesondere der beiden Weltkriege. Die auf allen Seiten zu beobachtende Mobilisierung der wirtschaftlichen, gesellschaftlichen und menschlichen Ressourcen in den am Krieg teilnehmenden Staaten habe gezeigt, dass es möglich ist, große Ziele (wie die Beseitigung der Armut, den Aufbau sozialer Sicherungssysteme sowie die massiven Investitionen des Staates in Bildung, Infrastruktur, Energiesektor und die Sicherstellung der Nahrungssicherheit) zu erreichen. Die Erfahrung der furchtbaren Konsequenzen beider Kriege habe aber auch die Einsicht in die Notwendigkeit der innenpolitischen Kompromisssuche und der gemäßigten Umverteilung bewirkt. Beides, die Erfahrung der Kraft der politischen Mobilisierbarkeit und der Kriege, habe zur
Entstehung von verantwortlichen und gemäßigten Staatlichkeiten beigetragen, wie wir sie heute in der westlichen Welt kennen würden.

In den Ländern außerhalb des Westens, so der Autor, habe es diese Erfahrung nie gegeben. Diese Staaten hätten keine vergleichbare Tradition der Mobilisierung ihrer Gesellschaften für große und existenzielle Kriege gehabt und seien in der Vergangenheit auch eher peripher in die beiden Weltkriege einbezogen worden. Die vor allem in Europa zu findende Bereitschaft der Bürger dem Staat Steuern zahlen, damit dieser große gesellschaftliche Ziele umsetze, sei in den meisten Staaten außerhalb Europas nicht zu finden. Der Staat werde als Herrschaftsinstrument etablierter oder revolutionärer Eliten begriffen, eine Wiederholung der positiven Erfahrung mit Staatlichkeit nach europäischem oder nordamerikanischem Beispiel sei unwahrscheinlich. Es sei angesichts der unterschiedlichen historischen Erfahrungen nicht wahrscheinlich und auch nicht wünschenswert, dass die nicht-europäischen Staaten den Weg der Europäer beschritten. Das Paradoxe sei dabei, dass, obwohl der Staat als Institution der Gesellschaft zur Bewältigung der großen Probleme nicht tauge, dieser weiterhin die primäre Organisationsform bleibe.

Um diesem Paradoxon zu entgehen, müsse versucht werden die Aufgaben moderner Staatlichkeit (also soziale Güter) an den real existierenden Staaten vorbei zu organisieren. Wie das im Einzelnen zu realisieren sei, wird vom Verfasser wiederholt angeschnitten, aber die Überlegungen bleiben sehr allgemein und weisen politisch kaum in eine klare Richtung. Was aber nach Lektüre des Buches übrig bleibt, ist die Aussage, dass fehlgeschlagene oder missbrauchte Staatlichkeit (Korruption, Kleptokratie, etc.) die Regel bleiben wird und auch nicht durch Entwicklungshilfe beseitigt werden kann. Folgt man Arjun Chowdhury, dann werden wir in absehbarer Zeit in einer derartigen Welt leben müssen. Das bedeutet, dass die internationale Ordnung der westlichen Welt (die immer auf der Vorherrschaft westlicher Demokratien und starker Staatlichkeit aufbaut) hier ihre Grenzen findet.

Es ist ein interessantes, nicht immer überzeugend argumentierendes Buch, welches aber eine Antwort auf Bardies Forderung nach dem Ende der westlichen „Arroganz" gibt: offensichtlich gibt es tiefere strukturelle Gründe dafür, dass die westliche Staatengemeinschaft in den letzten Jahrzehnten so erfolgreich war bei der Schaffung einer zumindest überregional wirksamen internationalen Ordnung, während andere Regionen aus ebenfalls strukturellen Gründen große Schwierigkeiten haben diesem Vorbild nachzuahmen. 
Geht man zu den eingangs genannten vier Fragen zurück, so lassen sich diese anhand der hier besprochenen Literatur wie folgt beantworten:

1. Die meisten Autoren stimmen darin überein, dass internationale Ordnung das Interesse von Staaten ausdrückt, die Unwägbarkeiten des Zustandes internationaler Anarchie abzumildern oder einzuhegen. Internationale Ordnung kann unterschiedliche Formen einnehmen, sie kann hegemonial oder imperial, sein, sie kann aber auch aus Arrangements der Großmächte bestehen, mit deren Hilfe sie bewaffnete Konflikte untereinander vermeiden oder eingrenzen wollen. Sie ist dann erfolgreich, wenn sie soziale Güter schafft (Sicherheit, Freihandel etc.) und auf einem breiten internationalen politischen Konsens beruht.

2. Fast alle Autoren gehen davon aus, dass die bislang gültige internationale Ordnung eine durch die USA gestiftete und angeführte Ordnung war und ist und einen wohlmeinenden Hegemon voraussetzt, der fähig und bereit ist für die Aufrechterhaltung dieser Ordnung Machtmittel (hard power, softpower) einzusetzen. Die konstitutiven Elemente sind eine Ordnung des internationalen Handels- und Finanzwesens sowie der Sicherheit vor Kriegen.

3. Der Zerfall der westlichen, liberalen internationalen Ordnung wird nach Ansicht der meisten Autoren einerseits durch den Aufstieg Chinas zur Weltmacht sowie die offene Herausforderung der Ordnung durch Russland und den Iran verursacht, andererseits aber auch durch den Rückzug der USA aus ihrer Führungsposition.

4. Die Konsequenzen des derzeitigen Ordnungsverfalls werden unterschiedlich eingeschätzt. Während die einen eher einen schrittweisen Wandel hin zu einer mehr pluralen Ordnung sehen, bei dem auch die Interessen anderer, nicht-westlicher Akteure stärker berücksichtigt werden müssen, sehen die meisten Autoren eher eine düstere Zukunft voraus, in der die internationalen Beziehungen erneut durch Machtkämpfe und strategische Rivalität gekennzeichnet seien. Allerdings gehen die meisten Autoren davon aus, dass westlich geprägte liberale internationale Ordnung fortexistieren kann, wenn sich die westlichen Staaten darüber einig sind und wenn sie akzeptieren, dass der universalistische Anspruch für absehbare Zeit nicht einzuhalten sein wird.

In einer Zeit, in der der Klimawandel und andere globale Probleme eigentlich erforderlich machen, dass die Staaten enger kooperieren, lassen diese Bücher ihre Leser nicht gerade in optimistischer Stimmung zurück.

\section{Literatur}

Bobbitt, Philip (2002): The Shield of Achilles. War, Peace and the Course of History. New York: Anchor Books

Bull, Hedley (1977): The Anarchical Society. A Study of Order in World Politics. Oxford University Press

Buzan, Barry (2004): From International to World Society? English School Theory and the Social Structure of Globalisation. Cambridge: Polity

Council on Foreign Relations (2019): Council of Councils Eighth Annual Conference. Insights from a Council of Councils Conference, May 2019, Report. New York: CFR, https://www.cfr. org/report/council-councils-eighth-annual-conference

Dunne, Timothy (1998): Inventing International Society. A History of the English School. Basingstoke: MacMillan

Fukuyama, Francis (2014): Political Order and Political Decay: From the Industrial Revolution to the Globalization of Democracy. New York: Farrar, Strauss \& Giroux

Ikenberry, G. John (2001): After Victory. Institutions, Strategic Restraint, and the Rebuilding if Order after Major Wars. Princeton: Princeton University Press

Ikenberry, G. John (2006): Liberal Order and Imperial Ambition. Cambridge/Malden, MA: Polity Press

Ikenberry, G. John (2011): Liberal Leviathan. The Origins, Crisis, and Transformation of the American World Order. Princeton/Oxford: Princeton University Press

Kissinger, Henry A. (1962): Großmachtdiplomatie. Von der Staatskunst Castlereaghs und Metternichs. Düsseldorf/Wien: Econ

Kissinger, Henry A. (1995) Diplomacy. New York u. a.: Simon \& Schuster

Kissinger, Henry A. (2014): World Order. Reflections on the Character of Nations and the Course of History. London und New York: Penguin/Allan Lane

Krause, Joachim (2007): Die internationale Ordnung in der Krise, Internationale Politik, 62 (7/8), 8-20

Krause, Joachim (2015): Englische Schule als IB-Theorie, in: Woyke, Wichard/Varwick, Johannes, Hrsg. (2015): Handwörterbuch Internationale Politik, 13. Auflage. Opladen und Toronto: Verlag Barbara Budrich, 65-70

Krause, Joachim (2017). Die neue Zeitenwende in den internationalen Beziehungen. Konsequenzen für deutsche und europäische Politik, Sirius - Zeitschrift für strategische Analysen, 1 (1), 3-24

Kundnani, Hans (2017): What is the Liberal International Order? Washington, D.C.: The German Marshall Fund (policy essay)

Mandelbaum, Michael (2016): Mission Failure: America and the World in the Post-Cold War Era. Oxford und New York: Oxford University Press

Watson, Adam (1992): The Evolution of International Society. London/New York: Routledge

Wight, Martin (1960): Why Is There No International Theory? International Relations, 2 (1), S. 35-48 